\title{
Evaluation of Chrysanthemum (Dendranthema grandiflora Tzvelev) for Desirable Horticultural Traits
}

\author{
Neelam Thakur ${ }^{1 *}$, Sujatha A. Nair ${ }^{1}$, Rajiv Kumar ${ }^{1}$, T. Usha Bharathi ${ }^{1}$, \\ M.V. Dhananjaya ${ }^{2}$ and R. Venugopalan ${ }^{3}$ \\ ${ }^{1}$ Division of Floriculture and Medicinal Crops, ${ }^{2}$ Division of Vegetables Crops, ${ }^{3}$ Division of \\ Social Sciences and Training, ICAR - Indian Institute of Horticultural Research, \\ Hesaraghatta Lake Post, Bengaluru 560 089, India \\ *Corresponding author
}

\section{A B S T R A C T}

\begin{tabular}{|c|}
\hline Keywords \\
\hline $\begin{array}{l}\text { Chrysanthemum, } \\
\text { Evaluation, } \\
\text { Genotypes, Floral } \\
\text { traits and shelf life }\end{array}$ \\
\hline Article Info \\
\hline $\begin{array}{l}\text { Accepted: } \\
\text { 06 July } 2018 \\
\text { Available Online: } \\
10 \text { August } 2018\end{array}$ \\
\hline
\end{tabular}

\begin{abstract}
An experiment was conducted to evaluate the performance of forty-nine chrysanthemum genotypes for desirable horticultural traits at ICAR-Indian Institute of Horticultural Research, Hesaraghatta Lake Post, Bengaluru from 2015-17. Significant variations were observed for various vegetative and flowering traits during both years. The results from the pooled data of two years showed that among the genotypes evaluated, maximum plant height was recorded in Arka Chandrakant $(69.59 \mathrm{~cm})$, maximum flower diameter in Arka Ravikiran $(9.00 \mathrm{~cm})$ and maximum weight of 100 flowers in Marigold $(925.04 \mathrm{~g})$. The genotype Roopanjali produced maximum number of flowers per plant (1199.17). Pusa Anmol recorded the minimum number of days to first flowering (60.48 days) while Roopanjali the longest period to first flowering (201.56 days). The genotypes with superior vegetative and floral traits desirable for the different segments of the floriculture industry like loose flower, cut flower and pot plant trade can be utilized in further improvement of chrysanthemum.
\end{abstract}

\section{Introduction}

Chrysanthemum (Dendranthema $x$ grandiflora Tzvelev) is one of the important floriculture crops in the cut flower, potted plant and herbaceous perennial markets of the world. The word chrysanthemum is derived from the Greek words 'chrysos' (gold) and 'anthemon' or 'anthos' (flower). It belongs to family Asteraceae (Anderson, 1987). It is believed to be native to the Northern hemisphere chiefly Europe and Asia and was believed to have been originated in China (Bose et al., 2002). Chrysanthemum inflorescences consist of central hermaphrodite disc florets (pistillate + staminate) and marginal ray florets (pistillate). The wide variations exhibited by a large number of cultivars with respect to growth habit, size, colour, and shape of blooms make the chrysanthemum flower suitable for various 
purposes such as borders, as cut flowers, pot plants, for garland making, hair decoration and for the exhibition.

Chrysanthemum covers 20090 ha area with production of $185240 \mathrm{MT}$ of loose flower and 14930 MT of cut flower in India during 20152016. Karnataka is the most prominent chrysanthemum growing state with an area of 5100 ha and production of 61200 MT of loose flower in 2014-2015 (Anonymous, 2018). Ease of cultivation, high returns, and increasing market demand are the main reasons for the popularity of this crop. As a photosensitive crop, chrysanthemum requires long days for vegetative growth and short days for flowering. Growth and flowering of chrysanthemum are very much influenced by light and temperature. Hence, the performance of genotypes varies with region, season and growing conditions. Selection as an important method of breeding is opted for identifying chrysanthemum genotypes with desirable horticultural traits for specific purposes and maximizing the production in commercial cultivation.

\section{Materials and Methods}

The present study was carried out at Division of Floriculture and Medicinal Crops, ICARIndian Institute of Horticultural Research, Hesaraghatta Lake Post, Bengaluru, India during 2016 (I year) and 2017 (II year). Data of both the years was pooled and analysed statistically. The experimental field was located at an altitude of 890 meters above MSL, $13^{0} 7^{\prime} \mathrm{N}$ latitude and $77^{0} 29^{\prime} \mathrm{E}$ longitudes. Forty-nine chrysanthemum genotypes were used for this study as mentioned in Table 1. The experiment was laid out in randomized block design with two replications.

Uniform terminal cuttings five to seven $\mathrm{cm}$ long were taken from healthy stock plants, dipped in IBA (Indole-3-butyric acid) 2500 ppm solution for 10 seconds and planted in plug trays containing the coco-peat medium for rooting. The rooted plants were transplanted on raised beds at a spacing of 30 x $30 \mathrm{~cm}$. The recommended dose of fertilizer was applied to the plants and irrigation was done through drip lines. Pinching was done 30 days after transplanting by removing the terminal portion of the plants. Regular monitoring of plants was done for disease infection and pest infestation. Prophylactic plant protection at the regular intervals was done during the course of the experiment to keep a check on diseases and pests. Observation was recorded on the vegetative traits like plant height $(\mathrm{cm})$ at the time of bud appearance, number of branches; and floral traits like days taken to bud appearance, days taken to first flowering, duration of flowering (days), number of flowers, flower diameter (cm) and flower weight $(\mathrm{g})$. The shelf life (days) of flowers was recorded by keeping the flowers in polythene bag (thickness 200 micron) at room temperature. Flower colour was recorded using RHS colour chart. The salient floral traits of the genotypes evaluated are detailed in Table 1.

\section{Results and Discussion}

Wide variation was recorded among the fortynine genotypes and these differed significantly for all the characters studied during both the years (Table 2). Chrysanthemum genotypes exhibit wide range of diversity with respect to growth habit, flower form and flower colour which can utilized for various purposes. These differences among genotypes provide variation for making selection in the crop improvement programme.

The genotypes recorded significant variation for plant height ranging from $16.07 \mathrm{~cm}$ to $69.61 \mathrm{~cm}$ with a mean value of $47.97 \mathrm{~cm}$. Arka Chandrakant $(69.61 \mathrm{~cm})$ was the tallest plants at first bud appearance stage whereas 
Arka Pink Star $(16.07 \mathrm{~cm})$ the shortest. In Chrysanthemum, taller plants are generally ideal for cut flower production, whereas medium to short with erect stem are preferred as compared to taller plants under open field condition to evade staking. Very short plants are ideal for bedding and pot plant production. The difference in vegetative attributes of different genotypes may be due to varied growth rate and their genetic makeup. Similar variation for plant height among genotypes was also observed by Madam et al., (2016), Kumar et al., (2014), Banerji et al., (2012) and Rao and Pratap (2006) in chrysanthemum genotypes.

Number of primary branches per plant is an important character which decides the canopy architecture of the plant. The genotype Sadbhavana $(17.47 \mathrm{~cm})$ had maximum number of primary branches per plant. Least number of primary branches per plant was recorded in Gulmohar (5.03). Differences observed in production of branches among the genotypes might be due to inherent genetic factors and similar results were reported by Srilatha et al., (2015), Kumar (2014), Gupta and Datta (2005) and Kulkarni and Reddy (2004).

Pusa Anmol (35.69) recorded the minimum days to first bud appearance while maximum number of days to first bud appearance (120.24) was recorded under Local Yellow Semi Double. These results are also corroborated with the findings of Yadav et al., (2014), Joshi et al., (2009), Swaroop et al., (2008) and Rao and Pratap (2006).

Days to first flowering gave an idea about the earliness of the genotypes which is useful to determine the flowering duration. Among all the genotypes, Pusa Anmol was the earliest and took 60.48 days for flowering followed by Ajay (75.32 days) and Vijay Kiran (76.69 days). The maximum numbers of days for flowering (201.56) was recorded in Roopanjali followed by Local Yellow Semi Double (189.57) and Autumn Joy (173.78) and are considered to be late flowering type. The variation for early and late blooming among chrysanthemum genotypes seems to be the genetically controlled character in the genotypes and similar observations were made by Negi et al., (2015), Yadav et al., (2014), Kumar and Poonam (2008) and Dilta et al., (2005).

The genotype Pusa Aditya flowered for a longer period of 45.53 days which was statistically at par with Arka Nilima (45.43 days) and also Rajat (44.62 days). The minimum flowering duration (21.10) was recorded in genotype Ajay. The variation for the duration of flowering among the genotypes can be attributed to differences in genetic makeup of the plants. Similar findings for variation in flowering duration among chrysanthemum genotypes have also been reported by Madam et al., (2016), Srilatha et al., (2015), Kumar (2014) and Kumar and Poonam (2006).

The number of flowers per plant varied from 44.06 to 1199.17 . The genotype Roopanjali (1199.17) had maximum number of flowers per plant followed by Basanti (858.62) and Aparajita (732.57) whereas Pusa Anmol (44.06) had minimum number of flowers.

The variation in number of flowers in these genotypes may be attributed to the inherent genetic and environmental factors. More number of branches per plant in some genotypes might be a reason for getting more number of flowers per plant. Similar variation for number of flowers per plants was reported earlier by was Reddy et al., (2016), Srilatha et al., (2015), Negi et al., (2015) and Punetha et al., (2011).

The flower size ranged from $2.5 \mathrm{~cm}$ to 9.00 $\mathrm{cm}$ in the forty-nine genotypes evaluated. 
Table.1 Flower colour and flower form of chrysanthemum genotypes

\begin{tabular}{|c|c|c|c|}
\hline Sl. No. & Genotype & Flower form & Flower colour \\
\hline 1. & Ajay & Decorative & 70B, Red-Purple Group, Fan 2 \\
\hline 2. & Anmol & Anemone & 3A, Yellow Group, Fan 1 \\
\hline 3. & Aparajita & Anemone & 9AYellow Group, Fan 1 \\
\hline 4. & Arka Chandrakant & Decorative & 155B, White Group, Fan 4 \\
\hline 5. & Arka Chandrika & Decorative & 155A, White Group, Fan 4 \\
\hline 6. & Arka Indira & Decorative & $\begin{array}{l}\text { Primary (upper)- 8A, Yellow Group, Fan 1; Secondary (lower)- 34A, } \\
\text { Orange-Red Group, Fan } 1\end{array}$ \\
\hline 7. & Arka Kirti & Double Korean & NN155A, White Group, Fan 4 \\
\hline 8. & Arka Nilima & Decorative & 72C, Red-Purple Group, Fan 2 \\
\hline 9. & Arka Pankaj & Decorative & 72D, Red-Purple Group, Fan 2 \\
\hline 10. & Arka Pink Star & Semi-double & 70B, Red-Purple, Fan 2 \\
\hline 11. & Arka Ravi & Korean & 22A, Yellow-Orange Group, Fan 1 \\
\hline 12. & Arka Ravikiran & Stellate & 181C, Greyed-Red Group, Fan 4 \\
\hline 13. & Arka Red Gold & Double Korean & N34A, Orange-Red Group, Fan 1 \\
\hline 14. & Arka Usha Kiran & Semi-double & 5B, Yellow Group, Fan 1 \\
\hline 15. & Arka Yellow Gold & Decorative & $\begin{array}{l}\text { Primary (upper)- 5C, Yellow Group, Fan 1; Secondary (streaks)- N34 A, } \\
\text { Orange-Red Group, Fan } 1\end{array}$ \\
\hline 16. & Arka Yellow Star & Decorative & 5A, Yellow Group, Fan 1 \\
\hline 17. & Autumn Joy & Decorative & 64A, Red-Purple Group, Fan 2 \\
\hline 18. & Basanti & Anemone & 3A, Yellow Group, Fan 1 \\
\hline 19. & CO.1 Chrysanthemum & Semi-double & 5A, Yellow Group, Fan 1 \\
\hline 20. & Coffee & Semi-double & N 34 B, Orange-Red Group, Fan 1 \\
\hline 21. & Fitonia & Single & 3A, Yellow Group, Fan 1 \\
\hline 22. & Flirt & Decorative & 71A, Red-Purple Group, Fan 2 \\
\hline 23. & Garden Beauty & Spoon & 53A, Red Group, Fan 1 \\
\hline 24. & Gulmohar & Double Korean & N78 B, Purple Group, Fan 2 \\
\hline 25. & Kargil & Spoon & $\begin{array}{l}\text { Primary (lower)- N155D, White Group, Fan 4, Secondary (margins)- 70B, } \\
\text { Red-Purple Group, Fan } 2\end{array}$ \\
\hline 26. & Lalpari & Single Korean & 53A, Red Group, Fan 1 \\
\hline 27. & Local Yellow Double & Pompon & 2A, Yellow Group, Fan 1 \\
\hline 28. & $\begin{array}{l}\text { Local Yellow Semi } \\
\text { Double }\end{array}$ & Pompon & 4A, Yellow Group, Fan 1 \\
\hline 29. & Marigold & Pompon & 5A, Yellow Group, Fan 1 \\
\hline 30. & Mother Teresa & Anemone & NN155D, White Group, Fan 4 \\
\hline 31. & Pink Cloud & Semi-double & 72 A, Red-Purple Group, Fan 2 \\
\hline 32. & Punjab Anuradha & Semi-double & 3A, Yellow Group, Fan 1 \\
\hline 33. & Pusa Aditya & Single & $\begin{array}{l}\text { Primary (upper)- 5A, Yellow Group, Fan 1; Secondary (lower)- } 34 \text { A, } \\
\text { Orange-Red Group, Fan1 }\end{array}$ \\
\hline 34. & Pusa Anmol & Decorative & 186B, Grey-Purple Group, Fan 4 \\
\hline 35. & Rajat & Stellate & NN155D, White Group, Fan 4 \\
\hline 36. & Ratlam Selection & Decorative & NN155D, White Group, Fan 4 \\
\hline 37. & Red Stone & Single & 53A, Red Group, Fan 1 \\
\hline 38. & Rekha & Single & $\begin{array}{l}\text { Primary (upper)- 34A, Orange-Red Group, Fan 1; Secondary (lower)- 5A, } \\
\text { Yellow Group, Fan1 }\end{array}$ \\
\hline 39 & Roopanjali & Single & 6A, Yellow Group, Fan 1 \\
\hline 40. & Sadbhavana & Double Korean & 53A, Red Group, Fan 1 \\
\hline 41. & Sharadmala & Double Korean & NN155D, White Group, Fan 4 \\
\hline 42. & Shyamal & Pompon & 14B, Yellow-Orange Group, Fan 1 \\
\hline 43. & Sunil & Decorative & N34A, Orange-Red Group, Fan 1 \\
\hline 44. & Vasanthika & Single & N34A, Orange-Red Group, Fan 1 \\
\hline 45. & Vijay Kiran & Double Korean & 9A, Yellow Group, Fan 1 \\
\hline 46. & White Andaman & Single & NN155D, White Group, Fan 4 \\
\hline 47. & White Dolley & Pompon & NN155A, White Group, Fan 4 \\
\hline 48. & Winter Queen & Spoon & 70B, Red-Purple Group, Fan 2 \\
\hline 49. & Yellow Delight & Pompon & 6B, Yellow Group, Fan 1 \\
\hline
\end{tabular}


Table.2 Vegetative, floral traits and keeping quality of chrysanthemum genotypes (pooled data of 2016 (I year) - 2017 (II year))

\begin{tabular}{|c|c|c|c|c|c|c|c|c|c|c|}
\hline Sl. No. & Genotype & $\begin{array}{l}\text { Plant } \\
\text { height } \\
(\mathrm{cm})\end{array}$ & $\begin{array}{l}\text { No. of } \\
\text { primary } \\
\text { branches }\end{array}$ & $\begin{array}{l}\text { Days taken to } \\
\text { bud } \\
\text { appearance }\end{array}$ & $\begin{array}{l}\text { Days taken } \\
\text { to first } \\
\text { flowering }\end{array}$ & $\begin{array}{l}\text { Duration of } \\
\text { flowering } \\
\text { (days) }\end{array}$ & $\begin{array}{l}\text { No. of } \\
\text { flowers }\end{array}$ & $\begin{array}{l}\text { Flower } \\
\text { diameter } \\
(\mathrm{cm})\end{array}$ & $\begin{array}{l}\text { Flower } \\
\text { weight } \\
\text { (g) }\end{array}$ & $\begin{array}{l}\text { Shelf } \\
\text { life } \\
\text { (days) }\end{array}$ \\
\hline 1. & Ajay & 36.72 & 7.76 & 44.93 & 75.32 & 21.10 & 56.20 & 4.77 & 167.76 & 3.00 \\
\hline 2. & Anmol & 49.17 & 7.02 & 62.17 & 142.88 & 40.33 & 203.61 & 3.73 & 88.00 & 2.00 \\
\hline 3. & Aparajita & 54.43 & 13.22 & 72.34 & 150.12 & 31.71 & 732.57 & 3.47 & 126.05 & 2.00 \\
\hline 4. & Arka Chandrakant & 69.61 & 8.00 & 82.55 & 125.59 & 35.51 & 142.40 & 7.98 & 809.72 & 5.25 \\
\hline 5. & Arka Chandrika & 62.41 & 9.31 & 65.29 & 103.88 & 39.32 & 305.06 & 6.40 & 350.50 & 5.25 \\
\hline 6. & Arka Indira & 47.51 & 10.15 & 60.81 & 111.95 & 42.68 & 161.43 & 8.19 & 647.08 & 5.00 \\
\hline 7. & Arka Kirti & 49.54 & 9.71 & 63.01 & 132.84 & 41.37 & 388.53 & 5.99 & 226.72 & 4.50 \\
\hline 8. & Arka Nilima & 61.20 & 7.82 & 76.52 & 153.65 & 45.43 & 250.11 & 6.74 & 366.09 & 5.00 \\
\hline 9. & Arka Pankaj & 53.24 & 10.54 & 70.92 & 142.57 & 41.07 & 287.33 & 7.75 & 349.91 & 5.00 \\
\hline 10. & Arka Pink Star & 16.07 & 14.80 & 44.25 & 78.64 & 23.25 & 322.67 & 3.00 & 82.29 & 2.00 \\
\hline 11. & Arka Ravi & 59.97 & 8.57 & 55.58 & 116.65 & 33.60 & 115.29 & 6.71 & 268.95 & 2.75 \\
\hline 12. & Arka Ravikiran & 61.91 & 10.75 & 79.42 & 150.02 & 34.64 & 264.75 & 9.00 & 341.41 & 4.00 \\
\hline 13. & Arka Red Gold & 50.00 & 10.59 & 70.96 & 143.97 & 31.80 & 305.92 & 6.61 & 260.15 & 4.00 \\
\hline 14. & Arka Usha Kiran & 47.99 & 8.82 & 66.05 & 125.26 & 41.04 & 314.32 & 6.49 & 223.11 & 6.00 \\
\hline 15. & Arka Yellow Gold & 59.81 & 8.43 & 66.95 & 153.92 & 37.55 & 312.08 & 7.77 & 299.37 & 5.00 \\
\hline 16. & Arka Yellow Star & 67.39 & 10.23 & 60.53 & 154.62 & 33.28 & 215.16 & 7.26 & 313.14 & 5.00 \\
\hline 17. & Autumn Joy & 45.94 & 12.30 & 58.51 & 100.14 & 36.40 & 385.96 & 7.86 & 284.01 & 3.00 \\
\hline 18. & Basanti & 55.04 & 16.82 & 63.34 & 151.05 & 34.36 & 858.62 & 4.47 & 151.12 & 3.00 \\
\hline 19. & $\begin{array}{l}\text { CO.1 } \\
\text { Chrysanthemum }\end{array}$ & 52.62 & 11.39 & 65.13 & 117.05 & 38.10 & 484.05 & 5.48 & 248.24 & 3.50 \\
\hline 20. & Coffee & 50.99 & 7.51 & 82.33 & 150.51 & 34.79 & 463.85 & 5.07 & 254.08 & 5.75 \\
\hline 21. & Fitonia & 43.96 & 8.77 & 69.00 & 150.33 & 23.45 & 574.36 & 3.49 & 100.38 & 3.00 \\
\hline 22. & Flirt & 51.97 & 8.01 & 73.18 & 149.60 & 40.89 & 140.48 & 7.89 & 505.07 & 4.00 \\
\hline 23. & Garden Beauty & 63.19 & 6.97 & 87.81 & 141.03 & 26.88 & 194.50 & 4.83 & 97.82 & 1.00 \\
\hline
\end{tabular}


Int.J.Curr.Microbiol.App.Sci (2018) 7(8): 565-574

\begin{tabular}{|c|c|c|c|c|c|c|c|c|c|c|}
\hline 24. & Gulmohar & 67.77 & 5.03 & 82.96 & 159.05 & 36.34 & 161.18 & 7.94 & 631.22 & 3.25 \\
\hline 25. & Kargil & 39.34 & 17.11 & 93.25 & 159.17 & 26.02 & 491.19 & 3.66 & 51.76 & 1.50 \\
\hline 26. & Lalpari & 47.00 & 9.34 & 74.23 & 156.50 & 27.47 & 580.31 & 3.06 & 52.73 & 2.00 \\
\hline 27. & $\begin{array}{l}\text { Local Yellow } \\
\text { Double }\end{array}$ & 48.62 & 7.27 & 73.77 & 133.37 & 37.92 & 330.41 & 6.52 & 465.96 & 5.75 \\
\hline 28. & $\begin{array}{l}\text { Local Yellow Semi } \\
\text { Double }\end{array}$ & 48.84 & 6.29 & 120.24 & 189.57 & 23.24 & 96.25 & 4.65 & 184.52 & 4.75 \\
\hline 29. & Marigold & 42.44 & 7.46 & 62.63 & 97.65 & 29.74 & 134.11 & 5.99 & 925.04 & 8.00 \\
\hline 30. & Mother Teresa & 33.09 & 17.07 & 64.08 & 138.35 & 33.85 & 425.09 & 3.63 & 95.06 & 3.00 \\
\hline 31. & Pink Cloud & 56.64 & 12.28 & 69.01 & 131.95 & 34.55 & 448.23 & 4.38 & 132.74 & 2.00 \\
\hline 32. & Punjab Anuradha & 55.44 & 11.00 & 65.35 & 119.22 & 32.00 & 410.71 & 5.48 & 261.08 & 3.50 \\
\hline 33. & Pusa Aditya & 39.95 & 11.65 & 64.94 & 139.53 & 45.53 & 441.14 & 5.05 & 112.97 & 1.00 \\
\hline 34. & Pusa Anmol & 23.81 & 7.73 & 35.69 & 60.48 & 23.20 & 44.06 & 4.55 & 123.37 & 2.00 \\
\hline 35. & Rajat & 51.69 & 9.24 & 64.51 & 137.76 & 44.62 & 420.77 & 8.12 & 415.54 & 5.25 \\
\hline 36. & Ratlam Selection & 63.46 & 11.27 & 70.25 & 125.89 & 34.25 & 315.04 & 8.13 & 796.52 & 5.25 \\
\hline 37. & Red Stone & 35.19 & 8.71 & 63.75 & 106.52 & 35.24 & 294.84 & 4.20 & 81.23 & 2.75 \\
\hline 38. & Rekha & 28.34 & 10.25 & 63.40 & 131.25 & 27.11 & 285.72 & 3.60 & 74.69 & 1.00 \\
\hline 39. & Roopanjali & 52.37 & 11.94 & 94.79 & 201.56 & 31.30 & 1199.17 & 2.50 & 57.14 & 1.25 \\
\hline 40. & Sadbhavana & 18.74 & 17.47 & 69.30 & 125.69 & 25.09 & 441.76 & 3.03 & 90.89 & 1.25 \\
\hline 41. & Sharadmala & 36.18 & 8.97 & 52.37 & 98.50 & 28.89 & 220.73 & 4.93 & 178.36 & 3.00 \\
\hline 42. & Shyamal & 54.49 & 12.48 & 81.19 & 159.25 & 30.27 & 496.19 & 3.53 & 174.45 & 4.00 \\
\hline 43. & Sunil & 50.10 & 10.43 & 68.47 & 138.96 & 35.50 & 355.18 & 6.15 & 238.40 & 4.00 \\
\hline 44. & Vasanthika & 51.79 & 10.83 & 69.90 & 137.31 & 42.31 & 231.78 & 4.93 & 110.18 & 2.75 \\
\hline 45. & VijayKiran & 30.48 & 8.95 & 58.62 & 76.69 & 31.51 & 159.36 & 4.26 & 93.55 & 2.00 \\
\hline 46. & White Andaman & 19.29 & 9.34 & 63.94 & 102.67 & 34.64 & 345.63 & 3.67 & 47.47 & 1.00 \\
\hline 47. & White Dolley & 24.73 & 8.15 & 65.14 & 112.42 & 29.98 & 218.73 & 3.97 & 222.08 & 2.50 \\
\hline 48. & Winter Queen & 51.89 & 11.45 & 91.55 & 173.77 & 33.39 & 466.98 & 5.81 & 64.67 & 1.00 \\
\hline \multirow[t]{6}{*}{49.} & Yellow Delight & 68.13 & 5.60 & 65.20 & 102.70 & 35.54 & 81.84 & 4.56 & 322.55 & 4.75 \\
\hline & Range & $\begin{array}{l}16.07- \\
69.61\end{array}$ & $5.03-17.47$ & $35.69-120.24$ & $60.48-201.56$ & $21.10-45.53$ & $\begin{array}{l}44.06- \\
1199.17\end{array}$ & $2.50-9.00$ & $\begin{array}{l}47.47- \\
925.04\end{array}$ & $\begin{array}{l}1.00- \\
8.00\end{array}$ \\
\hline & Mean & 47.97 & 10.10 & 69.18 & 130.35 & 33.84 & 338.38 & 5.45 & 256.43 & 3.42 \\
\hline & $\mathrm{SEm} \pm$ & 0.52 & 0.10 & 0.66 & 1.15 & 0.29 & 3.35 & 0.05 & 3.93 & 0.04 \\
\hline & C.D. $(p=0.05)$ & 1.02 & 0.19 & 1.31 & 2.27 & 0.57 & 6.63 & 0.10 & 7.77 & 0.07 \\
\hline & C.V. $(\%)$ & 1.52 & 1.33 & 1.36 & 1.24 & 1.19 & 1.40 & 1.36 & 2.17 & 1.50 \\
\hline
\end{tabular}


Table.3 Grouping of genotypes based on promising traits for different purposes

\begin{tabular}{|l|l|l|}
\hline Sl. No. & Genotype & Promising for use as \\
\hline 1. & $\begin{array}{l}\text { Arka Indira, Arka Nilima, Arka Pankaj, Arka Yellow } \\
\text { Gold, Arka Yellow Star, Ratlam Selection and Yellow } \\
\text { Delight }\end{array}$ & Cut flower \\
\hline 2. & $\begin{array}{l}\text { Arka Chandrika, Arka Usha Kiran, CO.1 Loose flower } \\
\text { Chrysanthemum, Marigold, Punjab Anuradha, } \\
\text { Sharadmala and Local Yellow Semi Double and Local } \\
\text { Yellow Double }\end{array}$ & \\
\hline 3. & $\begin{array}{l}\text { Arka Pink Star, Lalpari, Mother Teresa, Sadbhavana, } \\
\text { Vijay Kiran, White Andaman and White Dolley }\end{array}$ & $\begin{array}{l}\text { Pot Plant and bedding } \\
\text { purpose }\end{array}$ \\
\hline
\end{tabular}

Largest flower size $(9.00 \mathrm{~cm})$ was recorded in Arka Ravikiran. The flower size is minimum $(2.5 \mathrm{~cm})$ in Roopanjali. The variation in flower size might be due to genetic makeup of genotypes. This finding is also in accordance with Negi et al., (2015), Kumar et al., (2015), Kumar et al., (2014) and Gupta and Datta (2005) who reported significant difference in diameter different varieties of chrysanthemum.

The 100 flower weight also varied significantly, the maximum being recorded in the genotype Marigold (925.04 g) followed by Arka Chandrakant (809.72 g) and Ratlam Selection (796.52 g) whereas the genotype White Andaman $(47.47 \mathrm{~g})$ had minimum weight. This may be due to variation in number of ray florets. Similar observations were reported earlier by Reddy et al., (2016), Kumar (2014) and Swaroop et al., (2008).

The genotype Marigold (8.00 days) had maximum shelf life of the flowers followed by Arka Usha Kiran (6.00 days) and Coffee (5.75 days) whereas Pusa Aditya (1.00 day) minimum shelf life of the flowers. Variations in shelf life may be due to the different flower form, flower size and sensitivity of genotypes to ethylene. Significant differences among genotypes for various traits were also reported by Kumari et al., 2017 and Bhargav et al., 2018 in China aster.
RHS colour and flower form of the forty-nine chrysanthemum genotypes has been presented in Table 1. The forty-nine chrysanthemum genotypes were grouped into following colours, Yellow-Orange (Arka Ravi and Shyamal), White (Arka Chandrika, Arka Chandrakant, Arka Kirti, Rajat, White Dolley, White Andaman, Kargil, Ratlam Selection, Sharadmala and Mother Teresa), Yellow (Arka Indira, Arka Yellow Gold, arka Yellow star, Arka Usha Kiran, Pusa Aditya, Marigold, Basanti, Punjab Anuradha, CO.1 Chrysanthemum, Aparajita, Vijay Kiran, fitonia, Local Yellow semi Double, Anmol, Roopanjali, Yellow Delight and Local Yellow Double), Red-Purple (Arka Nilima, Arka Pankaj, Arka Pink Star, Flirt, Pink Cloud, Winter Queen, Ajay and Autumn Joy), Orange-Red (Vasantika, Coffee and Arka Red Gold), Greyed-Red (Arka Ravikiran), Red (Garden Beauty, Lalpari, Red Stone, Sadbhavana), Grey-Purple (Pusa Anmol), Purple (Gulmohar) and some genotypes having flowers with a dominant colour on top and tinge of another colour at the base of petals (Arka Indira, Arka Yellow Gold, Pusa Adiya and Kargil).

The forty-nine chrysanthemum genotypes showed different flower forms like Single Korean (Lalpari), Double Korean (Arka Ravi, Arka Kirti, Vijay Kiran, Gulmohar, Lalpari, Sharadmala, Sadbhavana and Arka Red 
Gold), Decorative (Arka Chandrika, Arka Chandrakant, Arka Indira, Arka Nilima, Arka Pankaj, Arka Yellow Gold, Arka Yellow Star, Flirt, Pusa Anmol, Ratlam Selection, Ajay, Autumn Joy and Sunil), Stellate (Arka Ravikiran and Rajat), Single (Vasantika, Pusa Aditya, White Andaman, Fitonia, Rekha, Red stone and Roopanjali), Semi-double (Arka Usha Kiran, Arka Pink Star, Punjab Anuradha, Pink Cloud and CO.1 Chrysanthemum), Pompon (Marigold, White Dolley, Local Yellow Semi Double, Yellow Delight, Local Yellow Double and Shyamal), Spoon (Garden Beauty, Kargil and Winter Queen), Anemone (Basanti, Aparajita, Anmol and Mother Teresa). Similar description of flower colour and flower type was also earlier recorded by Reddy et al., (2016), Poonam and Kumar (2008), Poonam and Kumar (2007) and Gupta and Datta (2005).

Arka Chandrakant, Yellow Delight, Gulmohar, Arka Yellow Star and Ratlam Selection are taller genotypes and Arka Pink Star, Sadbhavana, White Andaman, Pusa Anmol and White Dolley are shorter genotypes among the forty-nine chrysanthemum genotypes. Pusa Anmol, Ajay, Vijay Kiran, Arka Pink Star and Marigold are promising early flowering genotypes while Roopanjali, Local Yellow Semi Double, Winter Queen, Shyamal and Kargil are late flowering genotypes. Pusa Aditya, Arka Nilima, Rajat, Arka Indira and Vasantika are longer flowering duration genotypes whereas Ajay, Pusa Anmol, Local Yellow Semi Double, Arka Pink Star and Fitonia are shorter flowering duration genotypes. Based on these results the promising genotypes have been classified accordingly to their utility into the following Table 3.

Roopanjali, Basanti, Aparajita, Lalpari and Fitonia were highly floriferous among the forty-nine chrysanthemum genotypes. Arka
Ravikiran, Arka Indira, Ratlam Selection, Rajat and Arka Chandrakant are found promising for larger sized flower whereas Roopanjali, Arka Pink Star, Sadbhavana, Lalpari and Aparajita for small sized flower. Marigold, Arka Chandrakant, Ratlam Selection, Arka Indira and Gulmohar have higher 100 flower weight whereas White Andaman, Kargil, Lalpari, Roopanjali and Winter Queen have lower 100 flower weight among the forty-nine chrysanthemum genotypes. Marigold, Arka Usha Kiran, Coffee, Local Yellow Double and Arka Chandrika have longest shelf life among the chrysanthemum genotypes evaluated.

Considerable morphological variation was observed in all vegetative and flowering traits among the genotypes. These traits could be considered as useful selection criteria for further improvement in chrysanthemum.

\section{Acknowledgement}

We sincerely acknowledge the Director, ICAR-IIHR, Bengaluru for providing necessary facilities to conduct this study. The corresponding author is thankful to ICARIARI, New Delhi for awarding IARI fellowship during her Ph.D. programme.

\section{References}

Anderson, R.L. 1987. Reclassification of genus chrysanthemum. Hort. Science. 22: 313.

Anonymous. 2018. Ministry of Agriculture, Government of India. (https://www.indiastat.com/)

Banerji, B.K., Batra, A. and Dwivedi, A.K. 2012. Morphological and biochemical characterization of chrysanthemum. Journal of Horticultural Sciences, 7(1): 51-55.

Bhargav, V., Kumar, R., Rao, T.M., Bharathi, T.U., Dhananjaya, M.V., Kumar, S., 
Babu, K.S. and Kumari, P. 2018. Evaluation of China aster [Callistephus chinensis (L.) Nees] F1 hybrids and their parents for qualitative and quantitative Traits. Int.J.Curr. Microbiol.App.Sci. 7(02):1654 1661.

Bose, T.K., Yadav, L.P. and Pal, P. 2002. Chrysanthemum.

Commercial

Floriculture. $2^{\text {nd }}$ ed. Kolkata, Naya Prokash. pp. 463-602.

Dilta, B.S., Sharma, Y.D. and Verma, V.K. 2005. Evaluation of chrysanthemum cultivars under the sub-tropical region of Himachal Pradesh. Journal of Ornamental Horticulture, 8(2): 149151.

Gupta, V.N. and Datta, S.K. 2005. Morphological and chemical characterization of thirty small flowered chrysanthemum cultivars. Journal of Ornamental Horticulture, 8(2): 91-95.

Joshi, M., Verma, L.R. and Masu, M.M. 2009. Performance of different varieties of chrysanthemum in respect of growth, flowering and flower yield under north Gujarat condition. Asian Journal of Horticulture, 4(2): 292.

Kulkarni, B.S. and Reddy, B.S. 2004. Vegetative growth, flower yield and quantity of different chrysanthemum cultivars. Journal of Ornamental Horticulture, 7(3-4): 32-36.

Kumar, A. and Poonam. 2006. Garden Beauty, Winter Queen and Autumn Joy: new varieties of chrysanthemum for garden decoration. Journal of Research, 45(1 \& 2): 107.

Kumar, A. and Poonam. 2008. Yellow Delight: a new variety of chrysanthemum for cut flower production. Journal of Research, 45(1 \&2): 105.

Kumar, A., Dubey, P., Patanwar, M. and Sharma, R. 2015. Evaluation of chrysanthemum varieties for loose flower production in Chhattisgarh plains. Trends in Biosciences, 8(1): 175177.

Kumar, R. 2014. Evaluation of chrysanthemum genotypes for flowering traits under open grown condition. Hort Flora Research Spectrum, 3(4): 388-389.

Kumar, S., Kumar, M., Malik, S., Singh, M.K. and Kumar. S. 2014. Evaluation of chrysanthemum (Dendranthema grandiflora Tzvelev) genotypes using morphological characters under climatic conditions of western UP. Annals of Horticulture, 7(2): 162-165.

Kumari, P., Kumar, R., Rao, T.M., Bharathi, T.U., Dhananjaya, M.V. and Bhargav, V. 2017. Evaluation of China aster [Callistephus chinensis (1.) Nees] F1 hybrids and parents for growth, flower quality, yield and postharvest life. Int.J.Curr.Microbiol.App.Sci. $\quad$ 6(8): 1543-1549.

Madam, A.R., Jyothi, K.U., Vani, V.S., Reddy, A.R. and Ambati, R. 2016. Vegetative growth and flower yield as influenced by different chrysanthemum (Dendranthema grandiflora Tzvelev) cultivars in alfisols of coastal Andhra Pradesh. Annals of Horticulture, 9(1): 21-24.

Negi, R., Jarial, K., Kumar, S. and Dhiman, S.R. 2015. Evaluation of different cultivars of chrysanthemum suitable for low hill conditions of Himachal Pradesh. Journal of Hill Agriculture, 6(2): 144-146.

Poonam and Kumar, A. 2007. Garden Beautya promising chrysanthemum (Dendranthema grandiflora Tzvelev.) cultivar for garden decoration. Journal of Ornamental Horticulture, 10(3): 165168.

Poonam and Kumar, A. 2008. Autumn Joy- a new variety of chrysanthemum for garden decoration. Indian Journal of Plant Genetic Resources. 21(1): 77-80. 
Punetha, P., Rao, V.K. and Sharma, S.K. 2011. Evaluation of different chrysanthemum (Chrysanthemum morifolium) genotypes under mid hill conditions of Garhwal Himalaya. Indian Journal of Agricultural Sciences, 81(9):

Rao, A.M. and Pratap, M. 2006. Evaluation of varieties and variability studies in chrysanthemum (Dendrathema grandiflora Tzvelev.) Journal of Ornamental Horticulture, 9(2): 221223.

Reddy, A.M., Jyothi, U.K., Vani, V.S. and Reddy A.R. 2016. Evaluation of chrysanthemum (Dendranthema grandiflora Tzvelev.) cultivars for flower and post-harvest quality in alfisols of coastal Andhra Pradesh. Annals of Horticulture, 9(1): 4-8.

Srilatha, V., Kumar, K.S. and Kiran, Y.D. 2015. Evaluation of chrysanthemum
(Dendranthema grandiflora Tzvelev) varieties in the southern zone of Andhra Pradesh. Agricultural Science Digest, 35(2): 155-157.

Swaroop, K., Prasad, K.V. and Raju, D.V.S. 2008. Evaluation of chrysanthemum (Dendranthema grandiflora Tzvelev.) germplasm in winter season under Dehli conditions. Journal of Ornamental Horticulture, 11(1): 58-61.

Vetrivel, T. and Jawaharlal, M. 2014. Evaluation of chrysanthemum (Dendranthema grandiflora Tzvelev.) varieties for yield and quality under subtropical hills. Trends in Biosciences, 7(14): 1812-1815.

Yadav, A., Sharma, G. and Dubey, P. 2014. Flowering attributes of chrysanthemum varieties for loose flower production. Progressive Horticulture, 46(1): 168170.

\section{How to cite this article:}

Neelam Thakur, Sujatha A. Nair, Rajiv Kumar, T. Usha Bharathi, M.V. Dhananjaya and Venugopalan, R. 2018. Evaluation of Chrysanthemum (Dendranthema grandiflora Tzvelev) for Desirable Horticultural Traits. Int.J.Curr.Microbiol.App.Sci. 7(08): 565-574. doi: https://doi.org/10.20546/ijcmas.2018.708.062 